\title{
Mutations in mutT genes of Mycobacterium tuberculosis isolates of Beijing genotype
}

\begin{abstract}
Correspondence
Carlo Garzelli

garzelli@biomed.unipi.it
\end{abstract}

Received 25 July 2005

Accepted 9 January 2006

\author{
Nicoletta Lari, ${ }^{1}$ Laura Rindi, ${ }^{1}$ Daniela Bonanni, ${ }^{1}$ Enrico Tortoli ${ }^{2}$ \\ and Carlo Garzelli ${ }^{1}$
}
'Dipartimento di Patologia Sperimentale, Biotecnologie Mediche, Infettivologia ed Epidemiologia, Università di Pisa, I-56127 Pisa, Italy
${ }^{2}$ Centro Regionale di Riferimento per i Micobatteri, Laboratorio di Microbiologia e Virologia, Ospedale Careggi, I-50134 Firenze, Italy

\section{INTRODUCTION}

Molecular typing of Mycobacterium tuberculosis strains isolated in several countries in recent years has revealed that a family of strains known as 'Beijing' (or 'Beijing/W' or 'W-Beijing') is widespread around the world (Bifani et al., 2002; Filliol et al., 2003; Glynn et al., 2002). M. tuberculosis strains of Beijing genotype are mostly prevalent in Asia, but recent data suggest that they have spread to eastern Europe and Indo-China, being significantly more prevalent among younger patients than older patients in Vietnam (Anh et al., 2000). There is concern that the Beijing family may have a predilection for drug resistance, especially multidrug resistance (Glynn et al., 2002). In fact, the Beijing genotype of $M$. tuberculosis, with a higher prevalence of drug-resistance mutations than non-Beijing strains, has been identified in $40-50 \%$ of the clinical isolates studied in Russia during the last decade (Mokrousov et al., 2003).

In M. tuberculosis, resistance to anti-mycobacterial drugs is exclusively due to genomic mutations in specific genes (Ramaswamy \& Musser, 1998). With other bacteria, mutated phenotypes commonly result from defects in DNA repair (Horst et al., 1999), so it has been suggested that Beijing strains may have defective DNA repair systems, which would confer a mutator phenotype allowing an

Abbreviations: PGG, principal genotypic group; TB, tuberculosis. increased mutation rate, thus leading to a selective advantage during exposure to anti-mycobacterial drugs.

An in silico analysis has shown that most mismatch-repair systems commonly found in Escherichia coli (Mizrahi \& Andersen, 1998) are missing in M. tuberculosis, and only a number of putative genes encoding DNA repair enzymes, such as mutT, ogt, mutM and mutY, have been detected in the M. tuberculosis genome ( $\operatorname{Rad}$ et al., 2003). Analysis of strains representing different branches of the Beijing genotype has shown that the Beijing strains display unique missense alterations in putative mut genes, including two of the mutT type (the ORF Rv3908, designated mutT4 and mutT2) and ogt. These polymorphisms were found to be characteristic and unique to the Beijing phylogenetic lineage ( $\operatorname{Rad}$ et al., 2003).

The aim of this investigation was to study the variation in $m u t T$ genes in clinical isolates of Beijing $M$. tuberculosis to evaluate the contribution, if any, of mutT gene mutations to drug resistance.

\section{METHODS}

Clinical isolates. A total of $30 \mathrm{M}$. tuberculosis isolates of Beijing genotype, obtained from 2002 to 2004 from separate patients with tuberculosis (TB) hospitalized in Tuscany, Italy, were studied. Nineteen isolates were from patients with primary $\mathrm{TB}$ with no records of previous anti-TB therapy, two isolates were from patients 
previously treated with anti-TB drugs; previous $\mathrm{TB}$ and/or drug treatment was unknown for the remaining nine patients. A total of 24 non-Beijing strains resistant to multiple drugs and 28 fully susceptible non-Beijing strains isolated from 1993 to 2003, and during 2002 and 2003, in the same geographic area as the Beijing strains, were selected from our collection and used as controls. Assignment of isolates to the different genotypes was performed on the basis of the spoligotyping assay. All isolates were subjected to IS6110 RFLP typing and assigned to one of the three principal genotypic groups (PGGs) delineated by Sreevatsan et al. (1997) on the basis of the polymorphisms at codon 463 of the kat $G$ gene and codon 95 of gyrA gene (see below). The genotypes of the control isolates are reported in Table 1. Susceptibility of the isolates to isoniazid, rifampicin, ethambutol and pyrazinamide was determined by the radiometric BACTEC 460 TB system (Becton Dickinson) according to the proportion method. Drug resistance of Beijing isolates was compared with that of non-Beijing strains isolated in the same years and in the same geographic area as Beijing strains.

Molecular typing assays. Spoligotype analysis of isolates was performed as described by Kamerbeek et al. (1997), and the spoligotypes were compared to those contained in the SpolDB4 database (Brudey et al., 2006). IS6110-RFLP analysis of isolates was performed according to the standardized method described by van Embden et al. (1993). Polymorphisms at codon 463 of the kat $G$ gene and at codon 95 of gyrA gene of the isolates were evaluated by a real-time PCR assay, as previously reported (Rindi et al., 2004).

mutT genes mutations. Mutations in mutT genes were searched for by nucleotide sequencing using oligonucleotide primers pairs

\section{Table 1. Genotypes of non-Beijing strains}

Genotypes were defined according to the SpolDB4 database (Brudey et al., 2006). Abbreviations: CAS, central Asian; EAI, East African-Indian; LAM, Latino-American and Mediterranean. All non-Beijing isolates (resistant to multiple drugs or drug sensitive) displayed distinct IS6110-RFLP profiles, with the exception of the isolates of Bovis genotype showing the typical one-band pattern (data not shown).

\begin{tabular}{|lclc|}
\hline Drug resistance & No. tested & Genotype & PGG \\
\hline Multiple & 1 & Bovis & 1 \\
& 5 & LAM & 2 \\
& 1 & S & 2 \\
3 & Haarlem & 2 \\
& 1 & Haarlem & 3 \\
& 2 & T & 2 \\
None & 10 & T & 3 \\
& 1 & Undefined & 2 \\
3 & Bovis & 1 \\
& 3 & Africanum & 1 \\
6 & EAI & 1 \\
& 4 & CAS & 1 \\
4 & Haarlem & 2 \\
3 & LAM & 2 \\
1 & X & 2 \\
& 2 & T & 3 \\
1 & T & 2 \\
1 & Undefined & 1 \\
& & & \\
& & &
\end{tabular}

designed to amplify a 398 bp fragment of the mutT4 gene and a $675 \mathrm{bp}$ fragment of the mutT2 gene, both containing the mutation sites previously reported by Rad et al. (2003). The primers pairs were TAAGTCCTGGCCGACGATGG and CAACTCGATGTGCCCCTTGG for mutT4 gene, and GGCCATAAACGTCGGAAACTTG and CGCGTCCAGAAAACCATCGTAA for mutT2. PCR was performed in $0.5 \mathrm{ml}$ micro-centrifuge reaction tubes in a final volume of $50 \mu \mathrm{l}$, containing $50 \mathrm{mM}$ Tris/HCl $(\mathrm{pH} \mathrm{9.0),} 1.5 \mathrm{mM} \mathrm{MgCl}$, $15 \mathrm{mM}\left(\mathrm{NH}_{4}\right)_{2} \mathrm{SO}_{4}, 0 \cdot 1 \%$ Triton $\mathrm{X}-100,0 \cdot 25 \mu \mathrm{M}$ primers, $200 \mu \mathrm{M}$ dNTP, 1 U DyNAzyme EXT DNA polymerase and 30 ng DNA; after an initial denaturation step of $94^{\circ} \mathrm{C}$ for $3 \mathrm{~min}$, the amplification was performed with a PCR Express thermal cycler (Hybaid), set for $1 \mathrm{~min}$ at $94^{\circ} \mathrm{C}, 1 \mathrm{~min}$ at primer annealing temperatures $\left(64^{\circ} \mathrm{C}\right.$ for mutT4 and $65^{\circ} \mathrm{C}$ for mutT2), 2 min at $72^{\circ} \mathrm{C}$ for 30 cycles, followed by one final $4 \mathrm{~min}$ extension cycle at $72^{\circ} \mathrm{C}$. Direct sequencing of PCR products was carried out with a semi-automated apparatus (ALFexpress DNA sequencer; Pharmacia Biotech) using the Thermo Sequenase Cy5 dye terminator cycle sequencing kit (Amersham Pharmacia Biotech).

\section{RESULTS AND DISCUSSION}

\section{Molecular characteristics of Beijing isolates}

A total of 28 of the isolates assigned to the Beijing genotype showed the typical spoligotype pattern characterized by the deletion of spacers 1 to 34 in the direct repeat locus [octal number 000000000003771, share type (ST) 1]; the remaining 2 isolates showed deletions of spacers 1 to 36 and spacer 40 (isolate no. 838, octal number 000000000000731 , ST 406), and deletions of spacers 1 to 34 and spacers 38 to 42 (isolate no. 946, octal number 000000000003401, ST 940). All Beijing isolates belonged to PGG 1. By IS6110-RFLP analysis, the 30 Beijing isolates yielded a total of 20 distinct IS6110 patters; 15 isolates $(50 \%)$ occurred in 5 distinct clusters with identical IS6110 fingerprints, of these, 3 clusters contained 2 isolates, and 2 clusters contained 4 and 5 isolates (Fig. 1).

\section{Drug resistance and mutT mutations}

As shown in Table 2, 6 Beijing isolates $(20.0 \%)$ were resistant to isoniazid; such frequency, however, was not statistically different from that of isoniazid resistance (9.3\%) detected in 473 non-Beijing strains isolated in the same years and in the same geographic area. Resistance to rifampicin was not detected in any Beijing isolate; only one isolate displayed multiple resistances to isoniazid, ethambutol and pyrazinamide.

As shown in Fig. 1, nucleotide sequencing of mutT genes of Beijing isolates showed a base substitution at codon 48 of mutT4, consisting of a change of wild-type codon CGG to GGG resulting in the amino acid substitution of Arg by Gly, in 21 isolates; of these, 16 isolates also harboured a mutation at codon 58 of mutT2, a change of the wild-type codon GGA to CGA, resulting in an amino acid substitution of Gly by Arg. Resistance to one drug (isoniazid) was detected in 5 of the 21 isolates mutated in mutT4 (4 isolates also displayed the $m u t T 2$ mutation); 1 of the 9 isolates with wild-type $m u t T$ genes displayed multiple resistances. The association of 

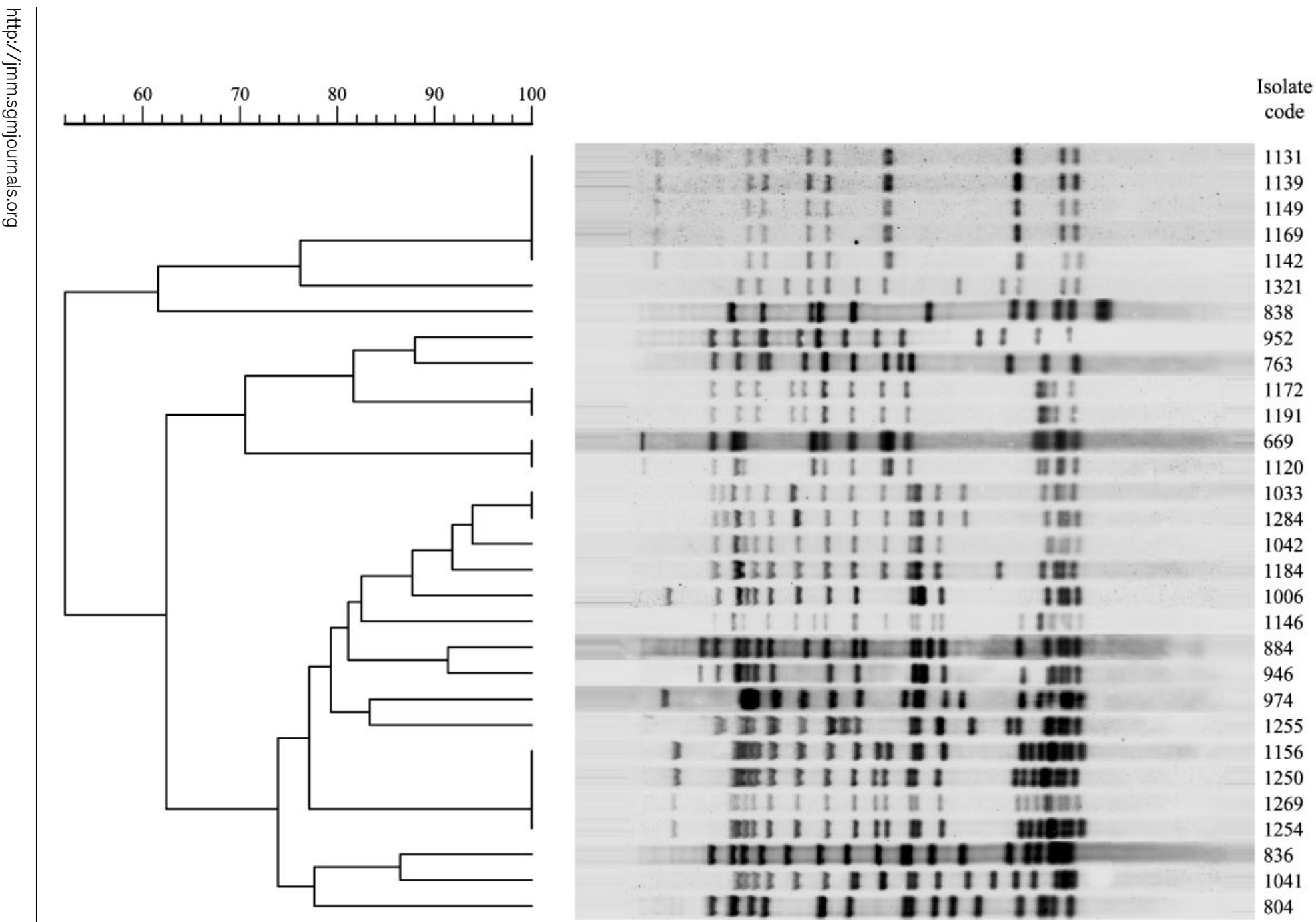

$\begin{array}{lccc}\text { Isolate } & \text { mutT4 } & \text { mutT2 } & \text { Drug } \\ \text { code } & \text { codon } 48 & \text { codon } 58 & \text { resistance }\end{array}$

Fig. 1. IS6110 fingerprints, mutT4 and mutT2 mutations, and drug resistance of 30 clinical isolates of M. tuberculosis of Beijing genotype. IS6110-RFLP patterns were compared and the dendrogram was constructed by using the UPGMA clustering method and the Dice coefficient by the Gelcompar 4.1 software package (Applied Maths). Isolate codes, wild-type (wt) or mutated codons of mutT4 and mutT2 genes, and drug resistance, expressed as number of drugs to which the isolate is resistant, are shown on the right of each RFLP panel. 
Table 2. Pattern of drug resistance of $M$. tuberculosis isolates of Beijing genotype compared to isolates of non-Beijing genotype

\begin{tabular}{|c|c|c|c|c|}
\hline \multirow[t]{3}{*}{ Drug $^{*}$} & \multicolumn{4}{|c|}{ Genotype } \\
\hline & \multicolumn{2}{|c|}{ Beijing } & \multicolumn{2}{|c|}{ Non-Beijing } \\
\hline & $\begin{array}{l}\text { Total } \\
\text { tested }\end{array}$ & $\begin{array}{l}\text { No. }(\%) \text { of drug } \\
\text { resistant isolates } \dagger\end{array}$ & $\begin{array}{l}\text { Total } \\
\text { tested }\end{array}$ & $\begin{array}{l}\text { No. (\%) of drug } \\
\text { resistant isolates } \dagger\end{array}$ \\
\hline INH & 30 & $6(20 \cdot 0)$ & 473 & $44(9 \cdot 3)$ \\
\hline RIF & 30 & $0(0)$ & 473 & $12(2 \cdot 5)$ \\
\hline ETH & 30 & $1(3 \cdot 3)$ & 464 & $11(2 \cdot 4)$ \\
\hline PZA & 30 & $1(3 \cdot 3)$ & 445 & $19(4 \cdot 3)$ \\
\hline Multiple drugs & - & $1(3 \cdot 3)$ & - & $11(2 \cdot 3)$ \\
\hline
\end{tabular}

${ }^{\star}$ ETH, ethambutol; INH, isoniazid; PZA, pyrazinamide; RIF, rifampicin.

$\dagger$ Determined by use of the radiometric BACTEC 460 TB system.

mutT4 and mutT2 mutations with drug resistance was not statistically significant ( $P=0.637$ by Fisher's exact test).

No mutation in mutT4 or mutT2 was found in any of 24 non-Beijing isolates resistant to multiple drugs, nor in any of 28 drug-susceptible non-Beijing strains.

\section{Conclusions}

This investigation, although carried out in a local setting where Beijing strains represent only a small proportion of the M. tuberculosis complex isolates (Lari et al., 2004, 2005), confirms that the polymorphism of the putative genes conferring a mutator phenotype is characteristic and unique to the Beijing phylogenetic lineage, as reported by Rad et al. (2003). Although the missense alterations detected in $m u t T$ genes do not appear to increase prevalence of resistance in the Beijing isolates of the present study, which are mostly primary isolates, it cannot be ruled out that the mutator phenotype might increase the rate of drug resistance mutations when strains are exposed to the selective pressure of anti-TB therapy. On this subject, further studies directly investigating the response of Beijing strains to anti-TB drugs are required.

\section{ACKNOWLEDGEMENTS}

This work was financially supported by MIUR (PRIN-2004) and, partly, by the Italian Istituto Superiore di Sanità (National Research Programme on AIDS-2003, ISS grant no. 50F.18).

\section{REFERENCES}

Anh, D. D., Borgdorff, M. W., Van, L. N., Lan, N. T., van Gorkom, T., Kremer, K. \& van Soolingen, D. (2000). Mycobacterium tuberculosis Beijing genotype emerging in Vietnam. Emerg Infect Dis 6, 302-305. Bifani, P. J., Mathema, B., Kurepina, N. E. \& Kreiswirth, B. N. (2002). Global dissemination of the Mycobacterium tuberculosis W-Beijing family strains. Trends Microbiol 10, 45-52.
Brudey, K., Driscoll, J. R., Rigouts, L. \& 63 other authors (2006). Mycobacterium tuberculosis complex genetic diversity: mining the fourth international spoligotyping database (SpolDB4) for classification, population genetics and epidemiology. BMC Microbiol 6, (in press).

Filliol, I., Driscoll, J. R., van Soolingen, D. \& 44 other authors (2003). Snapshot of moving and expanding clones of Mycobacterium tuberculosis and their global distribution assessed by spoligotyping in an international study. J Clin Microbiol 41, 1963-1970.

Glynn, J. R., Whiteley, J., Bifani, P. J., Kremer, K. \& van Soolingen, D. (2002). Worldwide occurrence of Beijing/W strains of Mycobacterium tuberculosis: a systematic review. Emerg Infect Dis 8, 843-849.

Horst, J. P., Wu, T. H. \& Marinus, M. G. (1999). Escherichia coli mutator genes. Trends Microbiol 7, 29-36.

Kamerbeek, J., Schouls, L., Kolk, A. \& 8 other authors (1997). Simultaneous detection and strain differentiation of Mycobacterium tuberculosis for diagnosis and epidemiology. J Clin Microbiol 35, 907-914.

Lari, N., Rindi, L., Bonanni, D., Tortoli, E. \& Garzelli, C. (2004). Beijing/W Mycobacterium tuberculosis in Italy. Emerg Infect Dis 10, 958-959.

Lari, N., Rindi, L., Sola, C., Bonanni, D., Rastogi, N., Tortoli, E. \& Garzelli, C. (2005). Genetic diversity, determined on the basis of katG463 and gyrA95 polymorphisms, spoligotyping, and IS6110 typing, of Mycobacterium tuberculosis complex isolates from Italy. J Clin Microbiol 43, 1617-1624.

Mizrahi, V. \& Andersen, S. J. (1998). DNA repair in Mycobacterium tuberculosis. What have we learnt from the genome sequence? Mol Microbiol 29, 1331-1339.

Mokrousov, I., Otten, T., Vyazovaya, A. \& 7 other authors (2003). PCR-based methodology for detecting multidrug-resistant strains of Mycobacterium tuberculosis Beijing family circulating in Russia. Eur J Clin Microbiol Infect Dis 22, 342-348.

Rad, M. E., Bifani, P., Martin, C. \& 8 other authors (2003). Mutations in putative mutator genes of Mycobacterium tuberculosis strains of the W-Beijing family. Emerg Infect Dis 9, 838-845.

Ramaswamy, S. \& Musser, J. M. (1998). Molecular genetic basis of antimicrobial agent resistance in Mycobacterium tuberculosis: 1998 update. Tuber Lung Dis 79, 3-29.

Rindi, L., Lari, N., Bonanni, D. \& Garzelli, C. (2004). Detection of Mycobacterium tuberculosis principal genetic groups by a duplex real-time PCR targeting the kat $G$ and gyrA genes. J Microbiol Methods 59, 283-287. 
Sreevatsan, S., Pan, X., Stockbauer, K., Connell, N., Kreiswirth, B., Whittam, T. \& Musser, J. (1997). Restricted structural gene polymorphism in the Mycobacterium tuberculosis complex indicates evolutionarily recent global dissemination. Proc Natl Acad Sci U S A 94, 9869-9874. van Embden, J. D. A., Cave, M. D., Crowford, J. T. \& 8 other authors (1993). Strain identification of Mycobacterium tuberculosis by DNA fingerprinting: recommendations for a standardized methodology. J Clin Microbiol 31, 406-409. 